\title{
Predictive risk factors for pneumothorax after transbronchial biopsy using endobronchial ultrasonography with a guide sheath
}

Yusuke Gotoh', Teppei Yamaguchi², Hiroshi Yatsuya ${ }^{3}$, Aki Ikeda' , Takuya Okamura', Yosuke Sakakibara', Takuma Ina', Yuri Maeda', Mariko Hirochi ${ }^{1}$, Hisashi Kako ${ }^{1}$, Yasuhiro Goto', Sumito Isogai ${ }^{1}$, Naoki Yamamoto ${ }^{4}$, Masashi Kondo ${ }^{1}$ and Kazuyoshi Imaizumi ${ }^{1 *}$

\begin{abstract}
Background: Pneumothorax is one complication of transbronchial biopsy (TBB) using endobronchial ultrasonography with a guide sheath (EBUS-GS-TBB). We sought to clarify the risk factors for pneumothorax after EBUS-GS-TBB under fluoroscopic guidance.
\end{abstract}

Methods: We retrospectively reviewed data from 916 patients who underwent EBUS-GS-TBB at Fujita Health University Hospital. We evaluated the following risk factors for pneumothorax after EBUS-GS-TBB: patient characteristics (sex, age, and pulmonary comorbidities); lesion data (location, size, existence of ground-glass opacities [GGOs], pleural involvement, computed tomography [CT] bronchus sign, visibility on fluoroscopy, and EBUS findings); final diagnosis; years of bronchoscopist experience; and guide sheath size. Univariate and multivariate logistic regression analyses were performed.

Results: Among the 916 patients, 30 (3.28\%) presented with pneumothorax. With a univariate analysis, factors that independently predisposed to pneumothorax included lesions containing GGOs, lesions in sagittal lung segments on fluoroscopy, lesions that were not visible on fluoroscopy, and infectious lesions. A univariate analysis also showed that lesions in the right upper lobe or left upper division, as well as malignant lesions, were less likely to lead to pneumothorax. Age, underlying pulmonary disease, CT bronchus sign, EBUS findings, bronchoscopist experience, and guide sheath size did not influence the incidence of pneumothorax. A multivariate analysis revealed that only lesions containing GGOs (odds ratio [OR] 6.47; 95\% confidence interval [CI] 2.13-19.6, $P=0.001$ ) and lesions in lung segments with a sagittal orientation on fluoroscopy (OR $2.47 ; 95 \% \mathrm{Cl} 1.09-5.58, P=0.029)$ were significant risk factors for EBUSGS-TBB-related pneumothorax.

Conclusions: EBUS-GS-TBB of lesions containing GGOs or lesions located in sagittal lung segments on fluoroscopy correlate with a higher pneumothorax risk.

Keywords: Bronchoscopy, Complication, Fluoroscopy, Pneumothorax, Endobronchial ultrasonography, Transbronchial biopsy

*Correspondence: jeanluc@fujita-hu.ac.jp

${ }^{1}$ Department of Respiratory Medicine, Fujita Health University School

of Medicine, 1-98 Dengakugakubo, Kutsukake-cho, Toyoake, Aichi

470-1192, Japan

Full list of author information is available at the end of the article

\section{Background}

Since chest computed tomography $(\mathrm{CT})$ screening for lung cancer has become a standard of care in routine clinical practice, there is an increasing need to diagnose small peripheral pulmonary lesions [1]. Transbronchial 
biopsy (TBB) using endobronchial ultrasound (EBUS) with a guide sheath (GS), referred to in this paper as EBUS-GS-TBB, is a recently introduced advanced bronchoscopic technique that has facilitated a higher diagnostic yield for small peripheral lung lesions compared with conventional TBB $[2,3]$. In general, TBB is a safe procedure for most patients. However, it sometimes leads to complications, including intrabronchial bleeding [4], infection [5], and pneumothorax [6], which can negatively affect the subsequent clinical course. Pneumothorax is one of the most common complications of TBB, with an incidence ranging from 1 to $4 \%$ [7]. Patients who develop pneumothorax may require chest tube drainage and prolonged hospitalization. In addition, for patients with respiratory insufficiency, pneumothorax may be serious or even fatal. Thus, preventing pneumothorax after TBB is vital. When using EBUS-GS-TBB, the precise location of the target lesion can be detected by ultrasound; however, this does not eliminate the possibility of pneumothorax [8]. Although previous studies have addressed the risk factors for pneumothorax after TBB with [9] or without [10] EBUS guidance, none have described the risk factors for pneumothorax after EBUSGS-TBB under fluoroscopic guidance. The present study aimed to evaluate the incidence of, and risk factors for, pneumothorax after EBUS-GS-TBB under fluoroscopic guidance.

\section{Methods}

\section{Patients and data acquisition}

This study was a single-center retrospective review. The medical records of patients who underwent EBUS-GSTBB for peripheral solitary lung lesions between August 2013 and March 2017 at Fujita Health University Hospital were examined. Peripheral pulmonary lesions were defined as lesions surrounded by lung parenchyma without direct visualization by bronchoscopy. Patients who underwent biopsy for $\geq 2$ separate lesions were excluded. We investigated the incidence of pneumothorax after EBUS-GS-TBB, sequential therapy for pneumothorax, and resulting outcomes. We also reviewed the following data of all patients: age; sex; smoking status; lung complications (emphysema, fibrosis, postoperative changes, and other lung diseases); steroid use; thin-section CT images of target lesions (size, presence of ground-glass opacities [GGOs], location, CT bronchus sign [3], presence of pleural involvement); EBUS findings (within, adjacent to, or outside) [2]; bronchoscopic diagnosis; and guide sheath size. This study was approved by the institutional review board of Fujita Health University (HM-19-017), and the study was performed in accordance with the principles of the Declaration of Helsinki.

\section{Bronchoscopic procedure}

All patients provided written informed consent before undergoing bronchoscopy. All patients underwent thinsection CT (0.5-mm slice thickness) within 1 month prior to the procedure. Each bronchoscopist evaluated the CT images and identified the bronchus and target lesion [3]. The responsible bronchus and the characteristics of target lesions were routinely confirmed by discussion between more than two bronchoscopists. A virtual navigation system was used in eight cases, all of which had a history of lobectomy (post-surgery). Pharyngeal local anesthesia with $2 \%$ lidocaine was administered prior to bronchoscopy. All patients received moderate sedation with midazolam at an individualized dose [11], and an additional intramuscular injection of $30 \mathrm{mg}$ of pethidine hydrochloride was administered to patients aged $<65$ years.

In total, 25 bronchoscopists performed EBUS-GS-TBB in the study period. The number of years of clinical experience ranged from 2 to 30 years (median, 5 years). We routinely perform bronchoscopy with the involvement of at least three bronchoscopists (one operator, one assistant, and one who monitors the patient's general condition). Five-hundred twenty-seven procedures (57.7\%) were performed by bronchoscopists with fewer than five years of experience in bronchoscopy.

EBUS-GS was performed using an endoscopic ultrasound system (EU-M30S, Olympus Co. Ltd., Tokyo, Japan) with a 20-MHz radial-type ultrasound probe (UMS20-20R, Olympus Co. Ltd.) according to the standard Kurimoto method [2]. We used two types of guide sheath kit (a large kit [K-203, 2.55-mm outer diameter guide sheath equipped with $1.9-\mathrm{mm}$ outer diameter biopsy forceps] or a small kit [K-201, 1.95-mm outer diameter guide sheath equipped with $1.5-\mathrm{mm}$ outer diameter biopsy forceps]) (Olympus Co. Ltd.) in combination with a UM-S20-20R probe. Flexible fiberoptic bronchoscopes used in this study were BF-260, BF-P260F, BF-P290, BF-1T260, and BF-1TQ290 (Olympus Co. Ltd.). We routinely obtained 12 biopsy samples with a small guide sheath kit (K201) and 6-9 samples with a large guide sheath kit (K203). TBB was routinely assisted by X-ray fluoroscopy (Canon Medical Systems, Tokyo, Japan). Blood pressure, heart rate, and oxygen saturation were continuously measured during bronchoscopy.

\section{Target lesion data analysis}

All target lesion imaging data were collected from CT images with a $0.5-\mathrm{mm}$ slice thickness generated using a non-enhanced multidetector CT system (Aquilion One Vision Edition; Toshiba Medical Systems, Tokyo, Japan) within 1 month before bronchoscopy. We collected data 
on the size, location, characteristics (GGOs vs. solid), $\mathrm{CT}$ bronchus sign $(\mathrm{A}-\mathrm{C}$, with $\mathrm{A}$ being the responsible bronchus that clearly reached the inside of the target lesion and $\mathrm{C}$ being no detectable responsible bronchus. If neither type A nor type $\mathrm{C}$ was concluded, the $\mathrm{CT}$ bronchus sign was categorized as type B) [3], existence of pleural involvement (contact or indentation), and GGOdominant nodules (consolidation-to-tumor ratio [CTR] of $<0.5$ ) designated as ground-glass nodules [GGNs]) [12]. In this study, we defined lung segments with a sagittal orientation on fluoroscopy (right: S2a, S3b, S5ab, S6a, S6c, S10a, S10c; left: S1+2b, S3b, S5ab, S6a, S6c, $\mathrm{S} 10 \mathrm{a}, \mathrm{S10c}$ ) as "lung segments in a sagittal direction." We focused on these segments because we could not easily recognize the pleural edge in the sagittal direction under fluoroscopic guidance. Thus, the positional relationship between the biopsy forceps and the pleura was difficult to recognize.

\section{Diagnosis and management of pneumothorax}

All patients underwent chest X-ray immediately following bronchoscopy and on the day after bronchoscopy. When pneumothorax was diagnosed, attending clinicians selected tube drainage or conservative management (observation) according to the extent of lung collapse and each patient's symptoms. If the apex of a collapsed lung was above the clavicle on chest X-ray (posteroanterior [PA] upright position) and the patient did not exhibit significant dyspnea, clinical observation was often selected; however, in some cases, chest drainage and prolonged hospitalization were used. Oxygen supplementation was administered based on the attending clinician's judgment.

\section{Statistical analysis}

To determine the risk factors for pneumothorax after EBUS-GS-TBB, we investigated the clinical factors related to pneumothorax after bronchoscopy, including age (> 75 years), sex, pulmonary comorbidities (severe emphysema and pulmonary fibrosis), lesion location, fluoroscopic view (not visible), lesion size (long diameter of $<20 \mathrm{~mm}$ ), GGNs (CTR of $>0.5$ ), CT bronchus sign (CT bronchus sign $\mathrm{B}$ or $\mathrm{C}$ ), pleural contact/indentation, EBUS findings (other than "within"), bronchoscopists' clinical experience, guide sheath size, and final diagnosis (malignancy and infectious disease). Associations between the variables of interest and the incidence of pneumothorax after EBUS-GS-TBB were first examined by univariate logistic regression and presented as odds ratios (ORs) and 95\% confidence intervals (CIs). Next, multivariate logistic regression for pneumothorax was performed using all variables that were used for the univariate analysis. A backward variable selection procedure was employed to obtain the final model. We presented mutually adjusted
ORs and 95\% CIs for pneumothorax after EBUS-GS-TBB for the final model. We also conducted stratified analyses according to lesion location; that is, lung segments in the sagittal direction, which are difficult to recognize under fluoroscopic guidance, or other subsegments. All statistical analyses were performed using JMP version 15.2 statistical software (SAS Institute Inc., Tokyo, Japan).

\section{Results}

\section{Patient and target lesion characteristics}

During the study period, 921 patients underwent EBUSGS-TBB. Five patients were excluded from the study because they underwent biopsy of $\geq 2$ lesions in different lung lobes. Thus, 916 patients were included in the study. Thirty patients (3.28\%) developed pneumothorax after EBUS-GS-TBB, which was resolved in 22 patients by observation. Eight patients required chest drainage, and four patients developed respiratory failure $\left(\mathrm{SpO}_{2}<90 \%\right)$ when pneumothorax occurred. All patients recovered without any surgical intervention or pleurodesis. None of the patients experienced pneumothorax recurrence. Patients' characteristics are shown in Table 1. The median age of patients was 72 years (range, 23-91 years). A total of 236 patients (25.7\%) had confluent or advanced destructive emphysema [13], and 129 patients (14.1\%) had pulmonary fibrosis. In 759 cases $(82.9 \%)$, we used a small guide sheath kit (K201).

Table 2 shows data on target lesions. The median lesion size was $23.13 \mathrm{~mm}$ (range, $5.5-112.8 \mathrm{~mm}$ ), and 52 lesions (5.6\%) were GGNs [12]. A total of 234 lesions (23.4\%) were present in sagittal lung subsegments. Lesions located in these segments were difficult to recognize under fluoroscopic guidance. The CT bronchus signs of 518 lesions $(56.5 \%)$ were positive, which indicated that the responsible bronchus clearly reached the inside of the target lesion [3]. EBUS findings of target lesions showed "within" in 558 cases (60.9\%). Final target lesion diagnoses were as follows: lung cancer or other malignancy (651 cases $[71.1 \%]$ ), infectious disease (56 cases [6.1\%]), other inflammatory disease (121 cases [13.2\%]), and other benign diseases (88 cases [9.6\%]). The total diagnostic yield of EBUS-GS-TBB in this study was $86.6 \%$.

\section{Predictive factors for pneumothorax}

The univariate logistic regression analysis revealed that lesions located in lung segments in a sagittal direction, lesions with GGN features, lesions that were not visible on fluoroscopy, and infectious lesions were prone to EBUS-GS-TBB-related pneumothorax. The univariate analysis also indicated that EBUS-GSTBB for lesions located in the right upper lobe or left upper division, as well as malignant lesions, were less likely to lead to pneumothorax (Table 3 ). The number 
Table 1 Characteristics of patients

\begin{tabular}{|c|c|c|c|}
\hline Characteristics & $\begin{array}{l}\text { Total } \\
\mathrm{n}=916\end{array}$ & $\begin{array}{l}\text { No pneumothorax } \\
\mathrm{n}=886\end{array}$ & $\begin{array}{l}\text { Pneumothorax } \\
n=30\end{array}$ \\
\hline \multicolumn{4}{|l|}{ Age } \\
\hline Median (IQR) & $72(65-77)$ & $71(65-77)$ & $71(60-74)$ \\
\hline$\geq 75$ years & $325(35.2 \%)$ & $313(35.3 \%)$ & $11(36.7 \%)$ \\
\hline \multicolumn{4}{|l|}{ Sex } \\
\hline Male & $609(66.5 \%)$ & $590(66.6 \%)$ & $19(63.3 \%)$ \\
\hline Female & 307 (33.5\%) & $296(33.4 \%)$ & $11(36.7 \%)$ \\
\hline \multicolumn{4}{|l|}{ Smoking status } \\
\hline Current & $230(25.1 \%)$ & $222(25.2 \%)$ & $8(26.7 \%)$ \\
\hline Ex & $628(68.6 \%)$ & $608(68.5 \%)$ & $13(43.3 \%)$ \\
\hline Never & $58(6.3 \%)$ & $56(6.3 \%)$ & $9(30.0 \%)$ \\
\hline \multicolumn{4}{|l|}{ Pulmonary co-morbidities } \\
\hline Emphysema* & $236(25.8 \%)$ & $227(25.6 \%)$ & $10(33.3 \%)$ \\
\hline Fibrosis & $129(14.1 \%)$ & $122(13.9 \%)$ & $7(23.3 \%)$ \\
\hline Post-surgery & $24(2.6 \%)$ & $24(2.7 \%)$ & $0(0 \%)$ \\
\hline Other lung diseases ${ }^{* *}$ & $88(9.6 \%)$ & $83(9.4 \%)$ & $5(16.7 \%)$ \\
\hline Steroid use & $35(3.8 \%)$ & 35 (3.9\%) & $0(0 \%)$ \\
\hline \multicolumn{4}{|l|}{ Guide sheath used } \\
\hline Large (outer diameter $2.55 \mathrm{~mm}$ ) & $157(17.1 \%)$ & $155(17.4 \%)$ & $3(10.0 \%)$ \\
\hline Small (outer diameter $1.95 \mathrm{~mm}$ ) & 759 (82.9\%) & 731 (82.5\%) & $27(90.0 \%)$ \\
\hline
\end{tabular}

* Confluent emphysema and advanced destructive emphysema [13]

** Other lung diseases, including bronchial asthma, sequelae of tuberculosis, bronchiectasis, sarcoidosis, pulmonary non-Mycobacterium tuberculosis infection, and pneumoconiosis

of years of clinical experience of each bronchoscopist did not influence the incidence of pneumothorax. The multivariate analysis revealed that GGN features (OR 6.47, 95\% CI 2.13-19.6, $P=0.001)$ and lesions located in sagittal lung segments (OR 2.47, 95\% CI 1.09-5.58, $P=0.029)$ were significantly associated with EBUSGS-TBB-related pneumothorax (Table 3).

Fluoroscopic recognition of target lesions depends on the imaging performance of the fluoroscopic system. For example, fluoroscopy with a $\mathrm{C}$-arm or conebeam CT could offer more recognizable images, even at lung segments in the sagittal direction. Thus, we further investigated whether the risk factors for EBUSGS-TBB-related pneumothorax changed when lesion location was excluded from the analysis. A multivariate analysis of patients whose lesions were located in sagittal lung subsegments $(\mathrm{n}=214)$ and patients whose lesions were present in other subsegments $(n=702)$ revealed that only GGNs were significant risk factors for EBUS-GS-TBB-related pneumothorax in both groups (OR 6.43, 95\% CI 1.89-21.89, $P=0.003$ and OR $5.02,95 \%$ CI $1.42-17.76, P=0.002$, respectively).

\section{Discussion}

The present study demonstrates that the risk factors for EBUS-GS-TBB-related pneumothorax are GGOs in target lesions and lesions located at lung subsegments in a sagittal direction.

GGO-dominant lesions were difficult to detect using fluoroscopy [14, 15]. EBUS recognition for GGO-dominant lesions is also difficult because ultrasound images of such lesions are sometimes vague or unrecognizable [12]. In fact, recent reports have evaluated the diagnostic yield of EBUS-GS-TBB for the diagnosis of GGNs, which ranged from 57 to $66 \%[12,16,17]$. This is lower than the diagnostic yield of EBUS-GS-TBB for lesions that present as solid nodules or with consolidation [12]. Izumo et al. reported the "blizzard sign or mixed-blizzard sign" as the specific EBUS finding for GGN lesions [14]. However, as these EBUS findings are subtle, it is difficult to confirm the precise location of GGNs using either EBUS or fluoroscopy. We speculate that difficulty in both fluoroscopic and EBUS recognition correlates with a significant increase in the occurrence of pneumothorax in GGN lesions. 
Table 2 Data on target lesions

\begin{tabular}{|c|c|c|c|}
\hline & $\begin{array}{l}\text { Total } \\
\mathrm{n}=916\end{array}$ & $\begin{array}{l}\text { No pneumothorax } \\
\mathrm{n}=886\end{array}$ & $\begin{array}{l}\text { Pneumothorax } \\
n=30\end{array}$ \\
\hline \multicolumn{4}{|l|}{ Lesion size } \\
\hline Median (IQR) mm & $23.08(17.1-32.8)$ & $23.13(17.1-32.8)$ & $20.38(17.4-33.2)$ \\
\hline$<20$ mm (long diameter) & $338(36.9 \%)$ & $325(36.7 \%)$ & $13(43.3 \%)$ \\
\hline \multicolumn{4}{|l|}{ Findings of thin-section CT } \\
\hline $\mathrm{GGN}^{*}$ & $53(5.8 \%)$ & $47(5.3 \%)$ & $6(20.0 \%)$ \\
\hline Solid & $863(94.2 \%)$ & $839(94.5 \%)$ & $24(80.0 \%)$ \\
\hline \multicolumn{4}{|l|}{ Location } \\
\hline Right upper lobe or left upper segments & $448(48.9 \%)$ & 440 (49.7\%) & $8(26.7 \%)$ \\
\hline Middle lobe or lingula & $127(13.9 \%)$ & $119(13.4 \%)$ & $8(26.7 \%)$ \\
\hline Lower lobe & $341(37.2 \%)$ & $327(36.9 \%)$ & $14(46.7 \%)$ \\
\hline Segments located in the sagittal direction ${ }^{* *}$ & $214(23.4 \%)$ & $201(22.7 \%)$ & $13(43.3 \%)$ \\
\hline \multicolumn{4}{|l|}{ CT bronchus sign ${ }^{* * *}$} \\
\hline A & $518(56.7 \%)$ & $503(56.7 \%)$ & $15(50.0 \%)$ \\
\hline B & $336(36.7 \%)$ & $323(36.5 \%)$ & $13(43.3 \%)$ \\
\hline C & $62(6.8 \%)$ & $60(6.8 \%)$ & $2(6.7 \%)$ \\
\hline \multicolumn{4}{|l|}{ Pleural involvement } \\
\hline Contact or indentation & $344(37.5 \%)$ & $333(37.6 \%)$ & $11(36.7 \%)$ \\
\hline No contact & $572(62.5 \%)$ & $553(62.4 \%)$ & $19(63.3 \%)$ \\
\hline \multicolumn{4}{|l|}{ Fluoroscopic view } \\
\hline Visible & $643(70.2 \%)$ & $627(70.8 \%)$ & $16(53.3 \%)$ \\
\hline Not visible & $273(29.8 \%)$ & $259(29.2 \%)$ & $14(46.7 \%)$ \\
\hline \multicolumn{4}{|l|}{ EBUS findings } \\
\hline Within & $558(60.9 \%)$ & $543(61.2 \%)$ & $15(53.3 \%)$ \\
\hline Adjacent to & $236(25.8 \%)$ & $227(25.6 \%)$ & $9(30.0 \%)$ \\
\hline Blizzard sign and mixed-blizzard sign & $97(10.5 \%)$ & $92(10.3 \%)$ & $5(16.7 \%)$ \\
\hline Outside & $25(2.7 \%)$ & $24(2.7 \%)$ & $1(3.3 \%)$ \\
\hline \multicolumn{4}{|l|}{ Bronchoscopic diagnosis } \\
\hline Malignancy & $647(70.6 \%)$ & $633(71.4 \%)$ & $14(46.7 \%)$ \\
\hline Infection & $55(6.0 \%)$ & $50(5.6 \%)$ & $5(16.7 \%)$ \\
\hline Organizing pneumonia & $45(4.9 \%)$ & $45(5.1 \%)$ & $0(0.0 \%)$ \\
\hline Other inflammatory lesions & $61(6.7 \%)$ & $60(6.7 \%)$ & $1(3.3 \%)$ \\
\hline Undiagnosed & $108(11.8 \%)$ & $98(12.1 \%)$ & $10(33.3 \%)$ \\
\hline \multicolumn{4}{|l|}{ Final diagnosis } \\
\hline Lung cancer or other malignancy & $651(71.1 \%)$ & $634(71.6 \%)$ & $17(56.7 \%)$ \\
\hline Infectious disease & $56(6.1 \%)$ & $51(5.7 \%)$ & $5(16.7 \%)$ \\
\hline Inflammatory disease & $121(13.2 \%)$ & $116(13.1 \%)$ & $5(16.7 \%)$ \\
\hline Other benign disease & $88(9.6 \%)$ & $85(9.6 \%)$ & $3(10.0 \%)$ \\
\hline
\end{tabular}

Abbreviations: CT, computed tomography; EBUS, endobronchial ultrasonography

${ }^{*}$ GGN, ground-glass-opacity-predominant nodule (consolidation-to-tumor ratio of <0.5)

** Lung segments that present with a sagittal orientation on fluoroscopy (right: S2a, S3b, S5ab, S6a, S6c, S10a, S10c; left: S1 + 2b, S3b, S5ab, S6a, S6c, S10a, S10c)

${ }_{* * *} \mathrm{CT}$ bronchus sign: type $\mathrm{A}$ (the responsible bronchus clearly reached the inside of the target lesion); type $\mathrm{C}$ (no bronchus could be detected in relation to the lesion); or type B (neither type A nor type C) [3]

Pneumothorax during TBB is caused by injury of the visceral pleura by biopsy forceps or bronchial brushes [18]. The tips of biopsy forceps or guide sheaths are difficult to recognize at subsegments in the sagittal direction when using fluoroscopy (anterior-posterior view). With conventional TBB without an EBUS guide, fluoroscopic guidance is essential to perform TBB of peripheral lung nodules. Although the EBUS system can directly detect peripheral lung lesions, it is impossible to perform biopsy with real-time ultrasound guidance in EBUS-GS-TBB. 
Table 3 Univariate and multivariate odds ratios (ORs) and 95\% confidence intervals (95\% Cls) of potential risk factors for EBUS-GS-TBBrelated pneumothorax

\begin{tabular}{|c|c|c|c|c|}
\hline & \multicolumn{3}{|c|}{ Univariate analysis } & \multirow{2}{*}{$\begin{array}{l}\text { Multivariate } \\
\text { analysis } \\
\text { P value }\end{array}$} \\
\hline & OR $(95 \% \mathrm{Cl})$ & $P$ value & OR $(95 \% \mathrm{Cl})$ & \\
\hline Age ( $\geq 75$ years) & $1.07(0.50-2.27)$ & 0.848 & & \\
\hline Sex (male) & $0.87(0.41-1.84)$ & 0.698 & & \\
\hline \multicolumn{5}{|l|}{ Pulmonary complication } \\
\hline Emphysema (severe emphysema*) & $1.46(0.67-3.17)$ & 0.395 & $2.13(0.47-2.12)$ & 0.074 \\
\hline Pulmonary fibrosis & $1.91(0.80-4.54)$ & 0.175 & & \\
\hline \multicolumn{5}{|l|}{ Lesion location } \\
\hline Upper lobe (Rt) or upper segment (Lt) & $0.37(0.16-0.84)$ & 0.015 & $0.38(0.14-1.01)$ & 0.053 \\
\hline Lingula or middle lobe & $2.39(1.04-5.49)$ & 0.052 & $1.46(0.45-2.99)$ & 0.358 \\
\hline Lower lobe & $1.49(0.72-3.10)$ & 0.337 & & \\
\hline Segments located in the sagittal direction ${ }^{* *}$ & $2.61(1.24-5.46)$ & 0.014 & $2.47(1.09-5.58)$ & 0.029 \\
\hline \multicolumn{5}{|l|}{ Lesion appearance } \\
\hline Long diameter (<20 mm) & $1.32(0.63-2.75)$ & 0.449 & & \\
\hline GGN*** (vs. solid lesion) & $4.46(1.74-11.44)$ & 0.006 & $6.47(2.13-19.6)$ & 0.001 \\
\hline CT bronchus sign B or $C^{\text {ref } X X}$ & $1.31(0.63-2.72)$ & 0.461 & & \\
\hline Pleural contact or indentation & $0.96(0.45-2.04)$ & 0.961 & & \\
\hline EBUS finding other than "within" & $1.39(0.67-2.87)$ & 0.450 & & \\
\hline EBUS finding "blizzard"/ "mixed-blizzard" & $1.96(0.73-5.26)$ & 0.195 & & \\
\hline Not visible on fluoroscopy & $2.11(1.01-4.40)$ & 0.043 & $1.46(0.65-3.28)$ & 0.358 \\
\hline \multicolumn{5}{|l|}{ Final diagnosis of target lesions } \\
\hline Malignancy & $0.46(0.22-0.96)$ & 0.042 & $0.64(0.66-3.67)$ & 0.312 \\
\hline Infectious disease & $3.34(1.23-9.11)$ & 0.029 & $3.28(0.99-10.87)$ & 0.051 \\
\hline \multicolumn{5}{|l|}{ Years of bronchoscopist experience } \\
\hline Fewer than 5 years & $1.27(0.60-2.73)$ & 0.510 & & \\
\hline \multicolumn{5}{|l|}{ Guide sheath } \\
\hline Large (diameter 2.6 mm) & $1.35(0.45-3.31)$ & 0.562 & & \\
\hline
\end{tabular}

${ }^{*}$ Confluent or advanced destructive emphysema [14]

** Right: S2a, S3b, S5a, S5b, S6a, S6c, S10a, S10c; left: S1 + 2b, S3b, S5a, S6a, S6c, S10a, S10c

${ }^{* * *}$ GGN: ground-glass-opacity-predominant nodule (consolidation-to-tumor ratio of $<0.5$ )

Abbreviations: CT, computed tomography; EBUS, endobronchial ultrasonography

Thus, fluoroscopy is essential to confirm the positional relationship of guide sheaths or biopsy forceps with the pleura. These data indicate that fluoroscopy during TBB is important, even with the EBUS guide, not only for a higher diagnostic yield, but also for procedural safety. More importantly, because most patients who undergo bronchoscopy are mildly sedated, it is difficult for the operator to recognize pleural pain, which may also be related to pneumothorax onset $[19,20]$. Thus, careful fluoroscopic observation during TBB is important, even with EBUS guidance.

Our study also showed that EBUS-GS-TBB for lesions in the upper lobe or upper division of the left upper lobe is unlikely to be accompanied by pneumothorax. With real-time fluoroscopic guidance, movement of lesions by breathing was less pronounced in the upper lung field compared with the lower lung field. Thus, lesions in the upper lobe and upper division may be easier to recognize during bronchoscopy under fluoroscopic guidance, which is beneficial for avoiding iatrogenic pneumothorax.

Huang et al. reported predictive risk factors for pneumothorax after EBUS-guided TBB [9]. They included pulmonary emphysema around target lesions and EBUS findings "adjacent to the lesion," both of which differ compared with our results. We speculate that the reason for these discrepancies is that Huang et al. did not use a guide sheath system in their study. The guide sheath system used in the present study is widely used because repeated biopsies from the planned point can be performed through the sheath placed on or near the target lesion. Use of a guide sheath and careful observation of EBUS findings could prevent unnecessary injury of emphysematous lung tissue. Another study showed that TBB from the upper lobes is significantly associated 
with post-TBB pneumothorax, which is different from our result [10]. However, this study included patients who underwent TBLB for diffuse lung lesions. Moreover, fluoroscopic guidance was not used in this study, and the incidence of post-TBB pneumothorax was higher compared with our study ( $6 \%$ vs. $3.28 \%$, respectively).

A recent study showed that even with newly developed robotic bronchoscopy, 3.7\% of patients are complicated with pneumothorax [21]. This report and our study suggest that it is difficult to completely prevent pneumothorax, even when TBB is performed using an advanced tool or technique. Thus, rapid and accurate diagnosis, as well as adequate and prompt therapy to treat pneumothorax, must be performed after TBB. Even with EBUS-GS support, TBB should be performed with caution in patients with identified risk factors. Some new modalities, such as cone-beam CT, may be beneficial for EBUS-GS-TBB of lesions at lung segments in the sagittal direction.

One limitation of our study is that it is a single-center study with a retrospective design. Bronchoscopy procedures, including biopsy, sedation, and fluoroscopy, vary between institutes. Thus, multi-institutional prospective studies are warranted in the future. Nevertheless, we believe our study reveals solid risk factors for pneumothorax after EBUS-GS-TBB, since these risk factors were determined from a large number of patients.

\section{Conclusions}

Our study shows that with EBUS-GS-TBB, we should be aware of the risk of pneumothorax when lesions are located in sagittal lung segments on fluoroscopy, because the positional relationship between biopsy forceps and the pleura is difficult to recognize, even under EBUS guidance. The risk of pneumothorax should also be considered when lesions present as GGOs, which are difficult to identify, even in combination with fluoroscopy and EBUS.

\footnotetext{
Abbreviations

Cl: Confidence interval; CT: Computed tomography; CTR: Consolidation-totumor ratio; EBUS-GS: Endobronchial ultrasonography with a guide sheath: GGN: Ground-glass nodule; IQR: Interquartile range; OR: Odds ratio; PA: Posteroanterior; TBB: Transbronchial biopsy.
}

\section{Acknowledgements}

We thank Ms. Naomi Maeda and Ms. Kaori Totani for their excellent secretarial support. We also thank Ms. Chieko Oka and Ms. Noriko Hiramatsu for their technical support. We thank Emily Woodhouse, PhD, from Edanz Group (https://jp.edanz.com/ac) for editing a draft of this manuscript.

\section{Authors' contributions}

Y. Gotoh, T. Y., and K. I. designed the work. Y. Gotoh, A. I., T. O., Y. S., T. I., Y. M., M. H., and H. K. contributed to data collection and analysis. Y. Gotoh, H. Y., Y. Goto, and N. Y. contributed to data interpretation. Y. Gotoh, T. Y., and K. I. drafted the manuscript. S. I. and M. K. revised the manuscript. The final version of the manuscript was approved by all authors. All authors read and approved the final manuscript.
Funding

None declared.

\section{Availability of data and materials}

The datasets generated and analyzed during the current study are available from the corresponding author on reasonable request.

\section{Declarations}

\section{Ethics approval and consent to participate}

This study was approved by the institutional review board of Fujita Health University (HM-19-017) and performed in accordance with the principles of the Declaration of Helsinki. The need for informed consent was waived by the Ethical Committee of Fujita Health University due to its retrospective nature.

\section{Consent for publication}

Not applicable.

\section{Competing interests}

The authors confirm that they have no competing interests to declare.

\section{Author details}

${ }^{1}$ Department of Respiratory Medicine, Fujita Health University School of Medicine, 1-98 Dengakugakubo, Kutsukake-cho, Toyoake, Aichi 470-1192, Japan. ${ }^{2}$ Department of Thoracic Oncology, Aichi Cancer Center Hospital, 1-1 Kanokoden, Chikusa-ku, Nagoya, Japan. ${ }^{3}$ Department of Public Health and Health Systems, Graduate School of Medicine, Nagoya University, 65 Tsurumai-cho, Nagoya, Japan. ${ }^{4}$ Department of Ophthalmology, Kanazawa Medical University, Ishikawa, Japan.

Received: 21 March 2021 Accepted: 17 May 2021

Published online: 29 May 2021

\section{References}

1. Mazzone PJ, Silvestri GA, Patel S, Kanne JP, Kinsinger LS, Wiener RS, Hoo GS, Detterbeck FC. Screening for lung cancer: CHEST Guideline and Expert Panel Report. Chest. 2018;153:954-85.

2. Kurimoto N, Miyazawa T, Okimasa S, Maeda A, Oiwa H, Miyazu Y, Murayama M. Endobronchial ultrasonography using a guide sheath increases the ability to diagnose peripheral pulmonary lesions endoscopically. Chest. 2004;126:959-65

3. Minezawa T, Okamura T, Yatsuya H, Yamamoto N, Morikawa S, Yamaguchi T, Morishita M, Niwa Y, Takeyama T, Mieno Y, Hoshino T, Uozu S, Goto Y, Hayashi M, Isogai S, Matsuo M, Nakanishi T, Hashimoto N, Okazawa M, Imaizumi K. Bronchus sign on thin-section computed tomography is a powerful predictive factor for successful transbronchial biopsy using endobronchial ultrasound with a guide sheath for small peripheral lung lesions: a retrospective observational study. BMC Med Imaging. 2015;15:21.

4. Folch EE, Mahajan AK, Oberg CL, Maldonado F, Toloza E, Krimsky WS, Oh S, Bowling MR, Benzaquen S, Kinsey CM, Mehta AC, Fernandez-Bussy S, Flandes J, Lau K, Krishna G, Nead MA, Herth F, Aragaki-Nakahodo AA, Barisione E, Bansal S, Zanchi D, Zgoda M, Lutz PO, Lentz RJ, Parks C, Salio M, Perret K, Keyes C, LeMense GP, Hinze JD, Majid A, Christensen M, Kazakov J, Labarca G, Waller E, Studnicka M, Teba CV, Khandhar SJ. Standardized definitions of bleeding after transbronchial lung biopsy: a Delphi consensus statement From the Nashville Working Group. Chest. 2020;158:393-400.

5. Souma T, Minezawa T, Yatsuya H, Okamura T, Yamatsuta K, Morikawa S, Horiguchi T, Maeda S, Goto Y, Hayashi M, Isogai S, Yamamoto N, Kondo M, Imaizumi K. Risk factors of infectious complications after endobronchial ultrasound-guided transbronchial biopsy. Chest. 2020;158:797-807.

6. Huang Y, Huang H, Li Q, Browning RF, Parrish S, Turner JF Jr, Zarogoulidis K, Kougioumtzi I, Dryllis G, Kioumis I, Pitsiou G, Papaiwannou A, Lampak S, Machairiotis N, Katsikogiannis N, Madesis A, Karaiskos T, Li Z, Zarogoulidis P. Transbronchial lung biopsy and pneumothorax. J Thorac Dis. 2014;6(Suppl 4):S443-7. 
7. Centonze CP, Davenport MS, White ES, Kazerooni EA. Routine chest radiography for the evaluation of pneumothorax following bronchoscopy. Acad Radiol. 2019;26:585-90.

8. Eom JS, Mok JH, Kim I, Lee MK, Lee G, Park H, Lee JW, Jeong YJ, Kim WY, Jo EJ, Kim MH, Lee K, Kim KU, Park HK. Radial probe endobronchial ultrasound using a guide sheath for peripheral lung lesions in beginners. BMC Pulm Med. 2018;18:137.

9. Huang CT, Ruan SY, Liao WY, Kuo YW, Lin CY, Tsai YJ, Ho CC, Yu CJ. Risk factors of pneumothorax after endobronchial ultrasound-guided transbronchial biopsy for peripheral lung lesions. PLoS ONE. 2012;7:e49125.

10. Herout V, Heroutova M, Merta Z, Cundrle I Jr, Brat K. Transbronchial biopsy from the upper pulmonary lobes is associated with increased risk of pneumothorax - a retrospective study. BMC Pulm Med. 2019:19:56.

11. Ogawa T, Imaizumi K, Hashimoto I, Shindo Y, Imai N, Uozu S, Shimokata T, Ito S, Hashimoto N, Sato M, Kondo M, Hasegawa Y. Prospective analysis of efficacy and safety of an individualized-midazolam-dosing protocol for sedation during prolonged bronchoscopy. Respir Investig. 2014;52:153-9.

12. Horiguchi T, Minezawa T, Okamura T, Sakakibara Y, Morikawa S, Gotoh Y, Souma T, Akao K, Watanabe T, Uozu S, Goto Y, Hayashi M, Isogai S, Imaizumi K. Endobronchial ultrasound-guided transbronchial biopsy for ground-glass opacity-predominant nodules in the lung periphery. Fujita Med J. 2018:4:11-6.

13. Lynch DA, Moore CM, Wilson C, Nevrekar D, Jennermann T, Humphries SM, Austin JHM, Grenier PA, Kauczor HU, Han MK, Regan EA, Make BJ, Bowler RP, Beaty TH, Curran-Everett D, Hokanson JE, Curtis JL, Silverman EK, Crapo JD. Genetic Epidemiology of COPD (COPDGene) Investigators. CT-based visual classification of emphysema: Association with Mortality in the COPD Gene Study. Radiology. 2018; 288: 859-66.

14. Izumo T, Sasada S, Chavez C, Matsumoto Y, Tsuchida T. Radial endobronchial ultrasound images for ground-glass opacity pulmonary lesions. Eur Respir J. 2015:45:1661-8.
15. Sasada S, Izumo T, Chavez C, Tsuchida T. Blizzard Sign as a specific endobronchial ultrasound image for ground glass opacity: a case report. Respir Med Case Rep. 2014;12:19-21.

16. Ikezawa Y, Sukoh N, Shinagawa N, Nakano K, Oizumi S, Nishimura M. Endobronchial ultrasonography with a guide sheath for pure or mixed ground-glass opacity lesions. Respiration. 2014;88:137-43.

17. Ikezawa Y, Shinagawa N, Sukoh N, Morimoto M, Kikuchi H, Watanabe M, Nakano K, Oizumi S, Nishimura M. Usefulness of endobronchial ultrasonography with a guide sheath and virtual bronchoscopic navigation for ground-glass opacity lesions. Ann Thorac Surg. 2017;103:470-5.

18. Maritato KC, Colon JA, Kergosien DH. Pneumothorax. Compend Contin Educ Vet. 2009:31:232-42.

19. Matsumoto T, Otsuka K, Kato R, Shimizu R, Otoshi T, Fujimoto D, Kawamura T, Tamai K, Nagata K, Otsuka K, Nakagawa A, Tomii K. Evaluation of discomfort and tolerability to bronchoscopy according to different sedation procedures with midazolam. Exp Ther Med. 2015;10:659-64.

20. Matot I, Kramer MR. Sedation in outpatient bronchoscopy. Respir Med. 2000:94:1145-53.

21. Chen AC, Pastis NJ Jr, Mahajan AK, Khandhar SJ, Simoff MJ, Machuzak MS, Cicenia J, Gildea TR, Silvestri GA. Robotic Bronchoscopy for peripheral pulmonary lesions: A Multicenter Pilot and Feasibility Study (BENEFIT). Chest. 2021;159:845-52.

\section{Publisher's Note}

Springer Nature remains neutral with regard to jurisdictional claims in published maps and institutional affiliations.
Ready to submit your research? Choose BMC and benefit from:

- fast, convenient online submission

- thorough peer review by experienced researchers in your field

- rapid publication on acceptance

- support for research data, including large and complex data types

- gold Open Access which fosters wider collaboration and increased citations

- maximum visibility for your research: over $100 \mathrm{M}$ website views per year

At BMC, research is always in progress.

Learn more biomedcentral.com/submissions 